\title{
Simulation of non-hydrostatic gravity wave propagation in the upper atmosphere
}

\author{
Y. Deng ${ }^{1}$ and A. J. Ridley ${ }^{2}$ \\ ${ }^{1}$ Department of Physics, University of Texas at Arlington, Arlington, Texas, USA \\ ${ }^{2}$ Department of AOSS, University of Michigan, Ann Arbor, Michigan, USA \\ Correspondence to: Y. Deng (yuedeng@uta.edu) and A. J. Ridley (ridley@umich.edu) \\ Received: 15 November 2013 - Revised: 12 March 2014 - Accepted: 26 March 2014 - Published: 24 April 2014
}

\begin{abstract}
The high-frequency and small horizontal scale gravity waves may be reflected and ducted in non-hydrostatic simulations, but usually propagate vertically in hydrostatic models. To examine gravity wave propagation, a preliminary study has been conducted with a global ionospherethermosphere model (GITM), which is a non-hydrostatic general circulation model for the upper atmosphere. GITM has been run regionally with a horizontal resolution of $0.2^{\circ}$ long $\times 0.2^{\circ}$ lat to resolve the gravity wave with wavelength of $250 \mathrm{~km}$. A cosine wave oscillation with amplitude of $30 \mathrm{~m} \mathrm{~s}^{-1}$ has been applied to the zonal wind at the low boundary, and both high-frequency and low-frequency waves have been tested. In the high-frequency case, the gravity wave stays below $200 \mathrm{~km}$, which indicates that the wave is reflected or ducted in propagation. The results are consistent with the theoretical analysis from the dispersion relationship when the wavelength is larger than the cutoff wavelength for the non-hydrostatic situation. However, the low-frequency wave propagates to the high altitudes during the whole simulation period, and the amplitude increases with height. This study shows that the non-hydrostatic model successfully reproduces the high-frequency gravity wave dissipation.
\end{abstract}

Keywords. Atmospheric composition and structure (thermosphere composition and chemistry)

\section{Introduction}

Many theoretical thermosphere/ionosphere models have been developed since 1970s, including thermospheric general circulation model (TGCM) (Dickinson et al., 1981), coupled thermosphere-ionosphere model (CTIM) (Fuller-Rowell and Rees, 1980) and their later variants. One common assumption used in these models is the hydrostatic equilibrium, under which the pressure gradient force is balanced with the gravity force in the vertical direction. This assumption is still used in many tropospheric global models (e.g., Yeager et al., 2006) and represents the large-scale atmosphere behavior very well. However, a comparative analysis of linearized dynamics with and without the hydrostatic approximation reveals its two effects on small-scale motions: it eliminates vertically propagating acoustic waves and significantly distorts properties of high-frequency gravity waves (Akmaev, 2011; Monin and Obukhov, 1958).

Upper atmospheric non-hydrostatic models (Chang and St.-Maurice, 1991; Deng et al., 2008, 2011) showed that sudden enhancements of Joule heating during geomagnetically disturbed periods generate intensive acoustic waves. Meanwhile, significant amounts of energy are deposited in the thermosphere through the acoustic waves propagating from the lower atmosphere (Hickey et al., 2001; Rind, 1977). It is very important for the atmospheric modeling to relax the hydrostatic assumption and allow the acoustic waves.

The nonhydrostatic dispersion relation for the gravity wave $(\mathrm{GW})$ is (Hines, 1960)

$m^{2}=\frac{k^{2} \omega_{\mathrm{B}}^{2}}{\omega^{2}}-\frac{1}{4 H^{2}}-k^{2}$,

where $k$ and $m$ are horizontal and vertical wave numbers; $\omega$ and $\omega_{\mathrm{B}}$ are the wave and buoyancy intrinsic frequencies; $H$ is the scale height. The last term on the right-hand side vanishes in the hydrostatic situation. The conditions for wave to be reflected and ducted $\left(m^{2}<0\right)$ are

$\lambda>\lambda_{\mathrm{n}}=4 \pi H \sqrt{\frac{\omega_{\mathrm{B}}^{2}}{\omega^{2}}-1}$ in the non-hydrostatic, 
Simulation domain $\left(0^{\circ}-20^{\circ} \mathrm{E}, 5^{\circ} \mathrm{S}-5^{\circ} \mathrm{N}\right)$

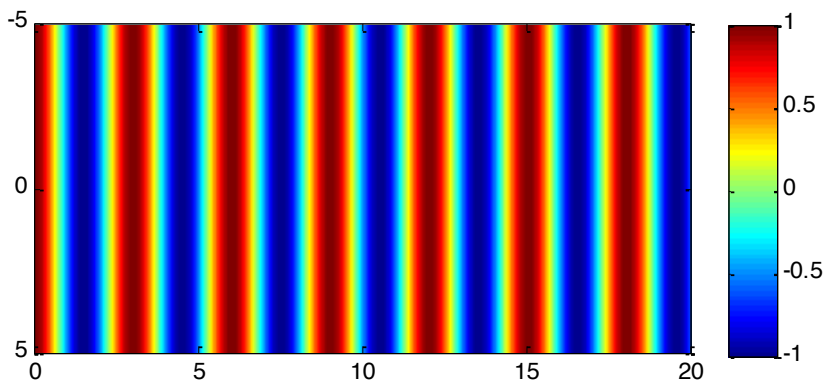

Fig. 1. Schematic drawing of the domain $\left(0-20^{\circ} \mathrm{E}, 5^{\circ} \mathrm{S}-5^{\circ} \mathrm{N}\right)$ for the regional simulation. A cosine wave oscillation with amplitude of $30 \mathrm{~m} \mathrm{~s}^{-1}$ has been applied to the zonal wind at the lower boundary at $100 \mathrm{~km}$ altitude $\left(V_{\text {east }}=30 \cdot \cos \left(\frac{2 \pi}{\lambda} x-\omega t\right)\right.$, where $\lambda$ is the wavelength, $x$ is the longitude, $\omega$ is the frequency and $t$ is the time).

$\lambda>\lambda_{\mathrm{h}}=4 \pi H \frac{\omega_{\mathrm{B}}}{\omega}$ in the hydrostatic cases,

where $\lambda$ is the horizontal wavelength of gravity wave, $\lambda_{\mathrm{n}}$ and $\lambda_{\mathrm{h}}$ are the cutoff wavelengths for the non-hydrostatic and hydrostatic cases, respectively (Akmaev, 2011). When $\lambda_{\mathrm{n}} \leq$ $\lambda \leq \lambda_{\mathrm{h}}$, the same wave behaves differently in hydrostatic and non-hydrostatic models. The gravity waves allowed to propagate vertically in a hydrostatic atmosphere may become internally reflected or ducted under the same conditions in a more realistic non-hydrostatic case, which indicates that the hydrostatic approximation potentially results in overestimation of the gravity wave vertical momentum flux and momentum deposition rates (Akmaev, 2011). For low-frequency waves $\left(\omega \ll \omega_{\mathrm{B}}\right)$, the difference between $\lambda_{\mathrm{n}}$ and $\lambda_{\mathrm{h}}$ is almost negligible. For high-frequency waves $\left(\omega \rightarrow \omega_{\mathrm{B}}\right), \lambda_{\mathrm{n}} \rightarrow 0$ and $\lambda_{\mathrm{h}} \rightarrow 4 \pi H$. Therefore, high-frequency waves with wavelengths of $\lambda \leq 4 \pi H$ should be treated non-hydrostatically. To be clear, Eq. (1) and the related discussion only apply to gravity waves and not acoustic waves, which can be triggered in the simulations when adding in the perturbation.

While the theoretical analysis indicates the significant difference between hydrostatic and non-hydrostatic simulations for the high-frequency small-scale gravity waves, to our knowledge very limited studies have been conducted on GCMs due to the limitation of models (Shutts and Vosper, 2011). In this study, a non-hydrostatic GCM has been used to investigate the behavior of gravity waves in the non-hydrostatic simulations, and the dissipation of highfrequency gravity wave has been reproduced in an upperatmospheric global model for the first time.

\section{Methodology}

The global ionosphere-thermosphere model (GITM) is a three-dimensional spherical code that models the Earth's thermosphere and ionosphere system using a stretched grid in latitude and altitude (Ridley et al., 2006). It solves for the neutral and ion densities, velocities and temperatures selfconsistently. The most significant difference between GITM and other upper-atmospheric models is that GITM does not assume a hydrostatic balance in the vertical direction. In addition, stretched grids in latitude and altitude are possible, and the number of grid points in each direction can be specified, so the resolution is flexible. GITM can also be run both globally and regionally. One limitation of GITM is the small time step (2-3 s), due to its explicit solver and the presence of vertically propagating acoustic waves in the solution (Deng et al., 2008), while other hydrostatic implicit GCMs may be run with much larger time steps $(2-5 \mathrm{~min})$.

In this study, GITM has been run regionally in a domain of $20^{\circ}$ long $\times 10^{\circ}$ lat centered at $10^{\circ} \mathrm{E}, 0^{\circ} \mathrm{N}$, as shown in Fig. 1. In order to resolve the high-frequency small-scale gravity waves with wavelength of $250 \mathrm{~km}$, the horizontal resolution is $0.2^{\circ}$ long $\times 0.2^{\circ}$ lat. The vertical resolution is onethird of the scale height. Open boundary conditions for the top boundary and specified boundary conditions for the lower boundary are used. On the left and right sides, a continuous gradient boundary condition is used on the densities and temperatures, while a continuous value is used on the velocity. To initialize the simulation, GITM was first run for $24 \mathrm{~h}$ during the September equinox, reaching a quasi-steady state under relatively quiet conditions. Then a cosine wave oscillation with amplitude of $30 \mathrm{~m} \mathrm{~s}^{-1}$ has been imposed to the zonal wind at the lower boundary at $100 \mathrm{~km}$ altitude ( $V_{\text {east }}=30 \cdot \cos \left(\frac{2 \pi}{\lambda} x-\omega t\right)$, where $\lambda$ is the wavelength, $x$ is the longitude, $\omega$ is the frequency and $t$ is the time). In this study, it is simply assumed that the disturbance only changes with longitude and is in phase along the latitude. To avoid broad-spectrum acoustic-gravity wave packets induced by a sudden switching on, the amplitude of perturbation at the lower boundary increases gradually from 0 to $30 \mathrm{~m} \mathrm{~s}^{-1}$ in $6 \mathrm{~min}$.

\section{Results and discussion}

In order to examine the frequency dependence of the wave propagation, three cases have been studied. This first case is the control case, in which no disturbance has been imposed. The second case is the high-frequency case, in which a high-frequency small-scale gravity wave $\left(\omega=0.75 \omega_{\mathrm{B}}\right.$, $\lambda=4 \pi H \sim 250 \mathrm{~km}$ ) has been applied to the zonal wind at lower boundary. For this frequency, the cutoff wavelength of hydrostatic and non-hydrostatic cases is $\lambda_{\mathrm{n}}=3.6 \pi H, \lambda_{\mathrm{h}}=$ $5.3 \pi H$. The wavelength of the gravity wave is between the two cutoff wavelengths $\left(\lambda_{\mathrm{n}}<\lambda<\lambda_{\mathrm{h}}\right)$. The third case is the low-frequency case, in which $\omega=0.25 \omega_{\mathrm{B}}, \lambda=4 \pi H$. For this frequency, the cutoff wavelength of hydrostatic and nonhydrostatic cases is $\lambda_{\mathrm{n}}=15.4 \pi H, \lambda_{\mathrm{h}}=16 \pi H$ and $\lambda<\lambda_{\mathrm{n}}<$ $\lambda_{\mathrm{h}}$. 
(a) $\mathrm{T}=3 \mathrm{~min}$

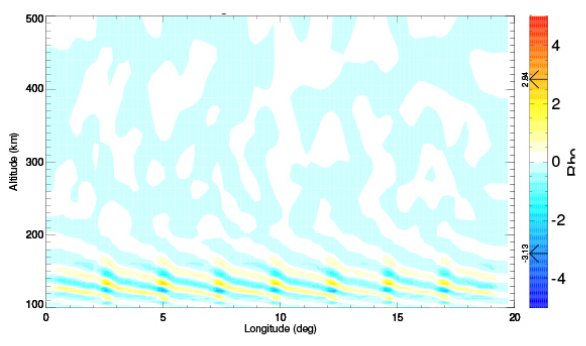

(c) $\mathrm{T}=12 \mathrm{~min}$

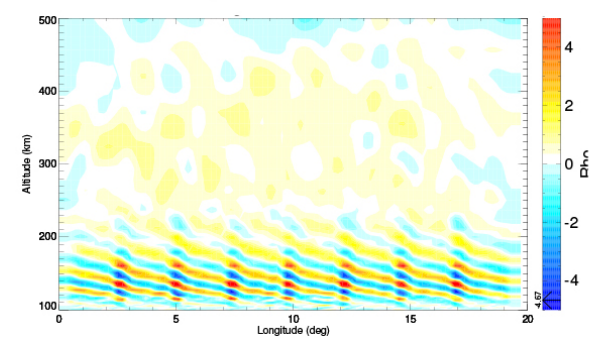

(b) $\mathrm{T}=6 \mathrm{~min}$

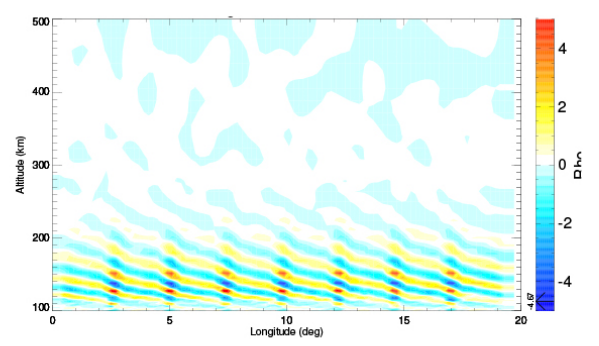

(d) $\mathrm{T}=18 \mathrm{~min}$

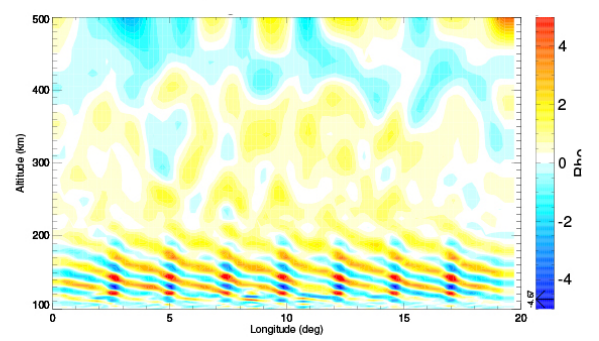

Fig. 2. Altitude-longitude distribution of neutral density percentage difference at equator $3 \mathrm{~min}(\mathbf{a}), 6 \mathrm{~min}(\mathbf{b}), 12 \mathrm{~min}(\mathbf{c})$ and $18 \mathrm{~min}(\mathbf{d})$ after adding in the high-frequency wave $\left(\omega=0.75 \omega_{\mathrm{B}}, \lambda=4 \pi H\right)$ oscillation to the zonal wind at the lower boundary. $\omega_{\mathrm{B}}$ is the buoyancy frequency, and $H$ is the scale height at the lower boundary.

(a) Vertical flux of horizontal momentum

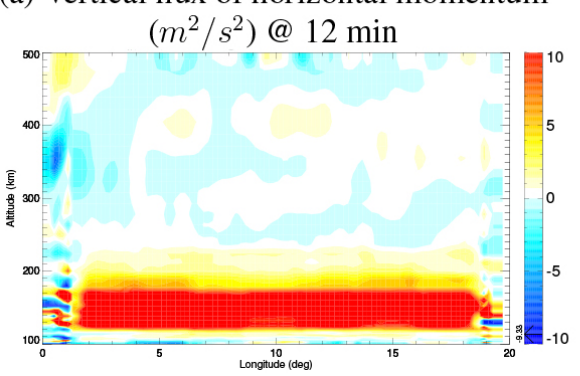

(b) Body force $\left(\mathrm{m} / \mathrm{s}^{2}\right) @ 12 \min$

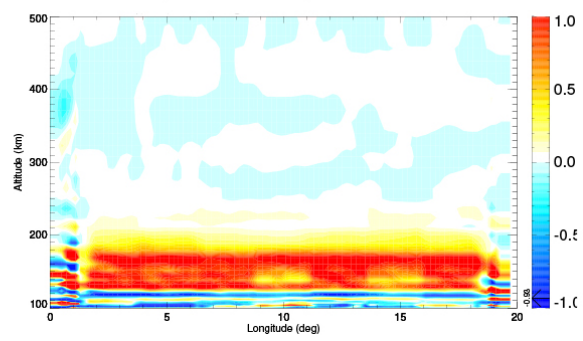

Fig. 3. Vertical flux of horizontal momentum (a) and body force (b) at $12 \mathrm{~min}$.

Figure 2 shows the percentage difference of neutral density between the high-frequency case and the control case at different times. The altitude-longitude distribution at equator has been displayed. The $x$ axis is longitude and the $y$ axis is altitude; $3 \mathrm{~min}$ after adding in the oscillation at the lower boundary, the wave propagates upward to $180 \mathrm{~km}$ altitude. At $6 \mathrm{~min}$, the wave stays at altitudes below $200 \mathrm{~km}$. After $12 \mathrm{~min}$, most disturbance is below $200 \mathrm{~km}$ and no significant wave has been propagated above that, which indicates that the wave got reflected or ducted below $200 \mathrm{~km}$ altitude. This result is consistent with the theoretical analysis from the dispersion relation as discussed in the introduction. It shows an example that our non-hydrostatic model is capable of reproducing the wave reflection or ducting, which has been predicted theoretically since the wavelength is larger than the cutoff wavelength for the non-hydrostatic case $\left(\lambda_{n} \leq \lambda\right)$. But the wavelength is still smaller than the cutoff wavelength in the hydrostatic case $\left(\lambda \leq \lambda_{h}\right)$, which means the wave may unrealistically propagate to higher altitude in hydrostatic model. It will be clearer when a similar run has been conducted in the hydrostatic model. However, to resolve the small-scale waves, sub-degree resolution is required, which is much higher than the resolution used in current hydrostatic GCM models.

Figure 3a shows the vertical flux of the horizontal momentum at $12 \mathrm{~min}$. The momentum flux decreases dramatically above $150 \mathrm{~km}$ and becomes almost negligible at $200 \mathrm{~km}$ and above. As discussed in Vadas and Liu (2009), the divergence of momentum flux is created by the dissipation of the smalland medium-scale gravity waves, which accelerates the background neutral wind. The thermospheric body force created from the gravity wave dissipation has been calculated using the formula from Vadas and Liu (2009). Figure 3b shows large body force below $200 \mathrm{~km}$. The magnitude can be more 

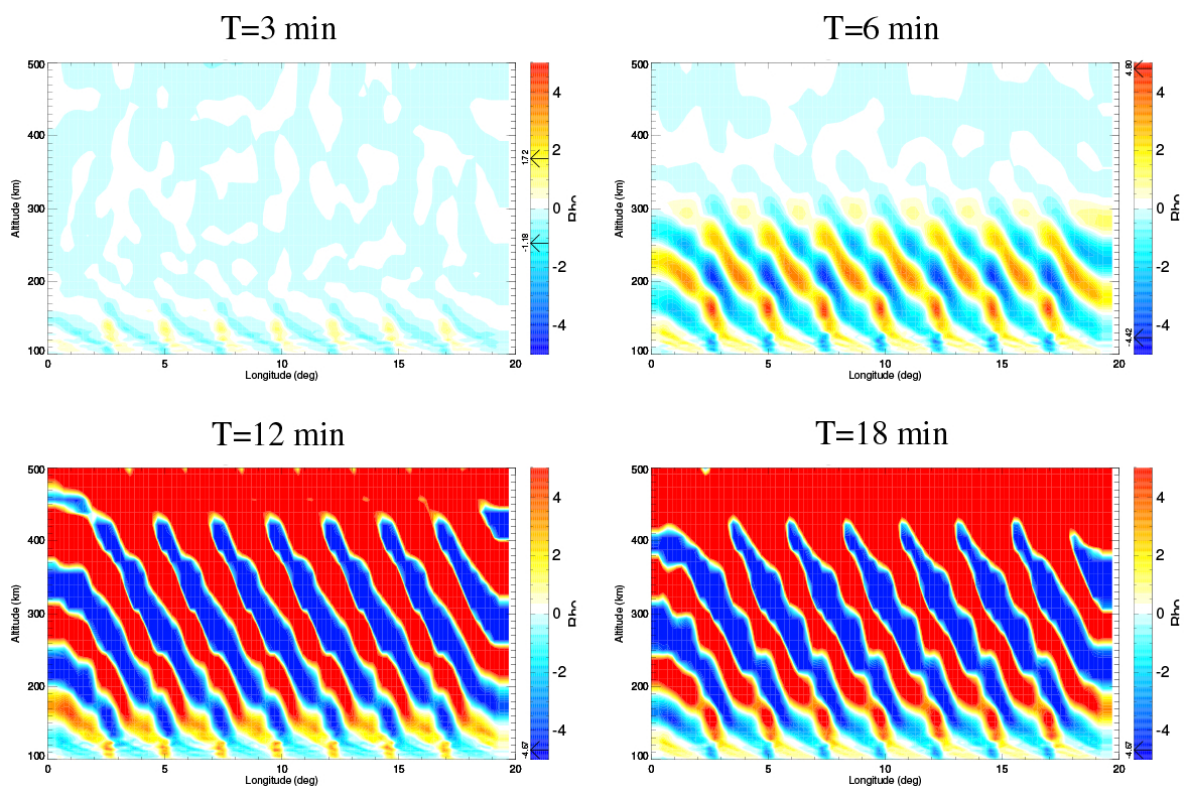

Fig. 4. The same as Fig. 2, but for the low-frequency wave $\left(\omega=0.25 \omega_{\mathrm{B}}, \lambda=4 \pi H\right)$.

than $1 \mathrm{~m} \mathrm{~s}^{-2}$, and the vertical depth is $\sim 80 \mathrm{~km}$, which are consistent with the results from the ray trace model (Vadas and Liu, 2009). The boundary conditions may strongly influence the variables close to the boundaries and cause something unrealistic. We will not emphasize the physical meaning of the features next to the boundaries.

Figure 4 shows the variation of neutral density when a low-frequency oscillation $\left(\omega=0.25 \omega_{\mathrm{B}}, \lambda=4 \pi H\right)$ is added in the lower boundary. The wavelength is smaller than cutoff frequency for both hydrostatic and non-hydrostatic cases $\left(\lambda<\lambda_{\mathrm{n}}<\lambda_{\mathrm{h}}\right)$, which indicates that the wave should be able to propagate in both hydrostatic and non-hydrostatic models. Indeed, the wave oscillation keeps propagating upward from the lower boundary during the whole simulation period as shown in Fig. 4. Compared to Fig. 2, the main difference is the large disturbance above $200 \mathrm{~km}$ attitude after $12 \mathrm{~min}$. The amplitude of the disturbance actually increases with altitude, which is consistent with the fact that the neutral density exponentially decreases with height and the perturbation energy is roughly conserved.

\section{Conclusions}

The analysis of the dispersion relationship indicates that high-frequency $\left(\omega \rightarrow \omega_{\mathrm{B}}\right)$ and small horizontal scale $(\lambda \leq$ $4 \pi H)$ gravity waves may be reflected and ducted in nonhydrostatic simulations, but usually propagate vertically in hydrostatic models. To investigate gravity wave dissipation in non-hydrostatic simulations, a preliminary study has been conducted with the global ionosphere-thermosphere model (GITM). GITM is a non-hydrostatic general circulation model for the upper atmosphere with a flexible spatial resolution. It can be run both globally and regionally. In this study, it has been run regionally in a domain of $20^{\circ}$ long $\times 10^{\circ}$ lat centered at $10^{\circ} \mathrm{E}, 0^{\circ} \mathrm{N}$ with the horizontal resolution of $0.2^{\circ}$ long $\times 0.2^{\circ}$ lat to resolve the high-frequency small-scale gravity wave with wavelength of $250 \mathrm{~km}$. A cosine wave oscillation with amplitude of $30 \mathrm{~m} \mathrm{~s}^{-1}$ has been applied to the zonal wind at the low boundary at $100 \mathrm{~km}$ altitude. The wavelength is close to $4 \pi H$, and two different frequencies have been tested including high-frequency $\left(\omega=0.75 \omega_{\mathrm{B}}\right)$ and lowfrequency $\left(\omega=0.25 \omega_{\mathrm{B}}\right)$ cases. In the high-frequency case, the gravity wave stays below $200 \mathrm{~km}$, which indicates that the wave is reflected or ducted in propagation. The results are consistent with the prediction from the dispersion relationship since the wavelength is larger than the cutoff wavelength in the non-hydrostatic situation $\left(\lambda_{\mathrm{n}} \leq \lambda\right)$. However, the lowfrequency wave propagates to the high altitudes during the whole simulation period, and the amplitude increases with height. This study shows that our non-hydrostatic model successfully reproduces the high-frequency small-scale gravity wave dissipation.

Acknowledgements. This research at the University of Texas at Arlington was supported by NSF through grant ATM0955629, by NASA through grant NNX13AD64G and NNX14AD46G and by AFOSR through Award 1210429.

Topical Editor C. Jacobi thanks R. A. Akmaev and one anonymous referee for their help in evaluating this paper. 


\section{References}

Akmaev, R. A.: Whole atmosphere modeling: Connecting terrestrial and space weather, Rev. Geophys., 49, RG4004, doi:10.1029/2011RG000364, 2011.

Chang, C. A. and St.-Maurice, J.-P.: Two-dimensional high-latitude thermospheric modeling - A comparison between moderate and extremely disturbed conditions, Can. J. Phys., 69, 1007-1031, 1991.

Deng, Y., Richmond, A. D., Ridley, A. J., and Liu, H.-L.: Assessment of the non-hydrostatic effect on the upper atmosphere using a general circulation model (GCM), Geophys. Res. Lett., 35, 1104, doi:10.1029/2007GL032182, 2008.

Deng, Y., Fuller-Rowell, T. J., Akmaev, R. A., and Ridley, A. J.: Impact of the altitudinal Joule heating distribution on the thermosphere, J. Geophys. Res.-Space, 116, A05313, doi:10.1029/2010JA016019, 2011.

Dickinson, R. E., Ridley, E. C., and Roble, R. G.: A three-dimensional, time-dependent general circulation model of the thermosphere, J. Geophys. Res., 86, 1499-1512, doi:10.1029/JA086iA03p01499, 1981.

Fuller-Rowell, T. J. and Rees, D.: A three-dimensional, timedependent, global model of the thermosphere, J. Atmos. Sci., 37, 2545-2567, 1980.
Hickey, M. P., Schubert, G., and Walterscheid, R. L.: Acoustic wave heating of the thermosphere, J. Geophys. Res., 106, 21543 21548, doi:10.1029/2001JA000036, 2001.

Hines, C. O.: Internal atmospheric gravity waves at ionospheric heights, Can. J. Phys., 38, 1441, doi:10.1139/p60-150, 1960.

Monin, A. S. and Obukhov, A. M.: Small oscillations of the atmosphere and adaptation of meteorological fields, Izv. Akad. Nauk SSSR, Ser. Geofiz., 1958.

Ridley, A. J., Deng, Y., and Tóth, G.: The global ionosphere thermosphere model, J. Atmos. Sol.-Terr., 68, 839-864, doi:10.1016/j.jastp.2006.01.008, 2006.

Rind, D.: Heating of the lower thermosphere by the dissipation of acoustic waves, J. Atmos. Terr. Phys., 39, 445-456, 1977.

Shutts, G. J. and Vosper, S. B.: Stratospheric gravity waves revealed in NWP model forecasts, Q. J. Roy. Meteorol. Soc., 137, 303 317, doi:10.1002/qj.763, 2011.

Vadas, S. L. and Liu, H.-L.: Generation of large-scale gravity waves and neutral winds in the thermosphere from the dissipation of convectively generated gravity waves, J. Geophys. Res.-Space, 114, A10310, doi:10.1029/2009JA014108, 2009.

Yeager, S. G., Large, W. G., and Hack, J. J.: The Low-Resolution CCSM3, J. Climate, 19, 2545, doi:10.1175/JCLI3744.1, 2006. 